توليد جوانههاى نابجا از كثت بافت ريزنمونهى دمبرك جغنندرقند در روش باززايى مستقيم

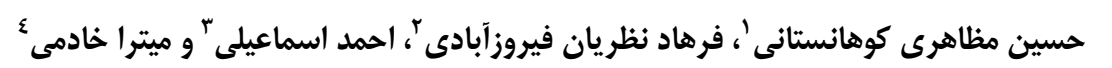

l، ب و F- دانش آموخته كارشناسى ارشد، دانشيار و دانشجوى دكترى، دانشكده كشاورزى، دانشخاه لرستان

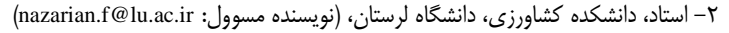
ت

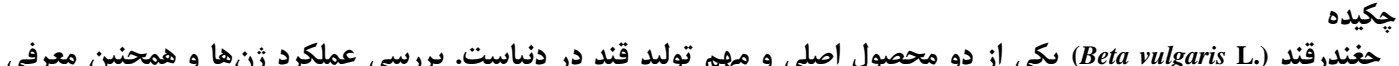

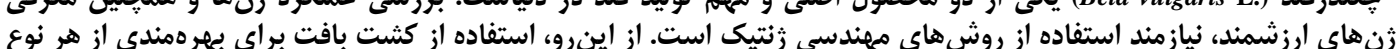

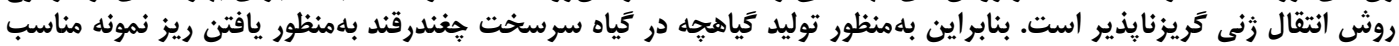

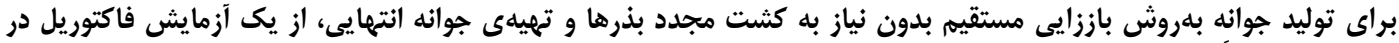

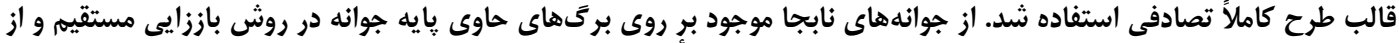

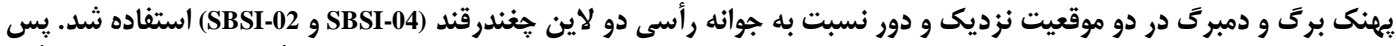

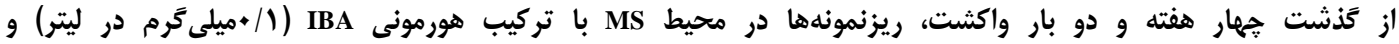

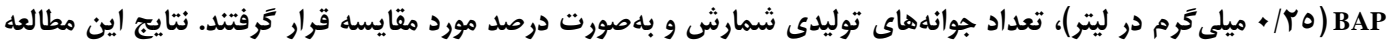

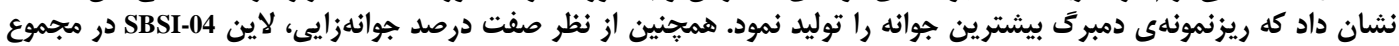

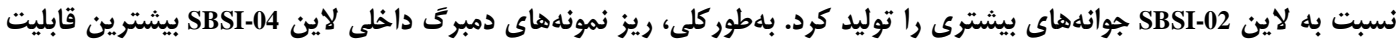

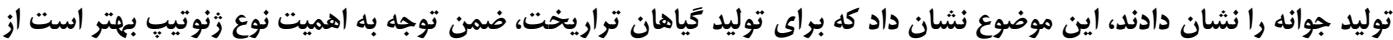
كثت بافت اين ريزنمونه و تراريزش أن أن استفاده شود. نوان

وازههاى كليدى: باززايى مستقيه، تراريزش، دمبرك، ريزنمونه، هورمونهاى رشد

هايدارى زنتيكى بالاترى برخوردار هستند (سا) لذا باززايى

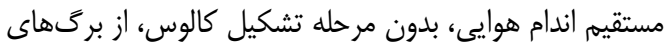

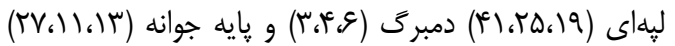

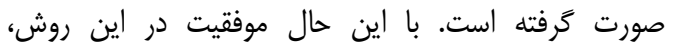

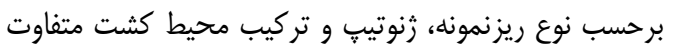

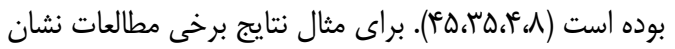

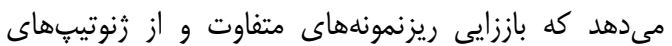

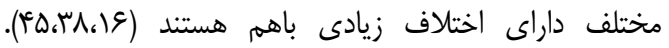

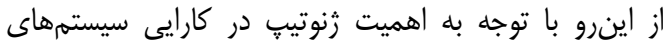

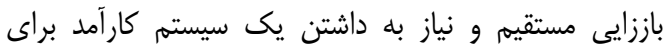

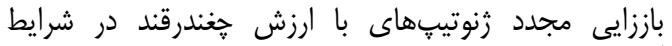

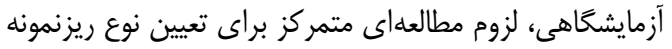

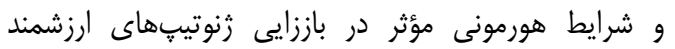

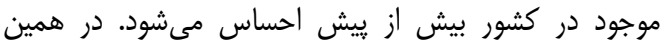

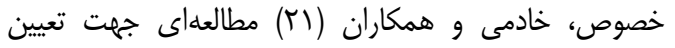

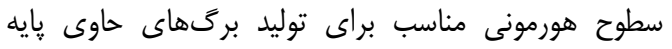

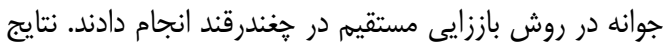

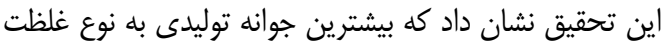

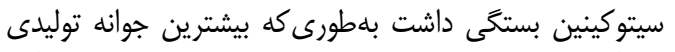

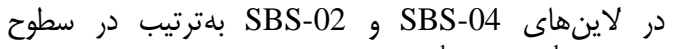

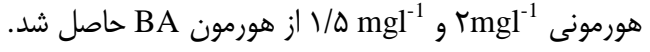

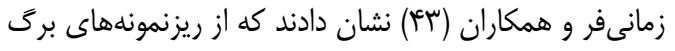

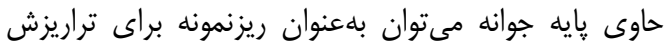

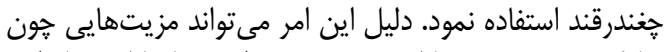

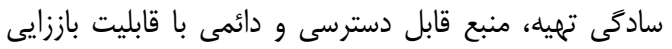

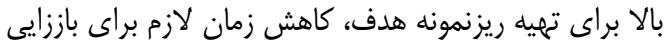

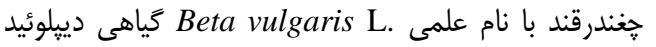
(Chenopediaceae) (Yn=|人)

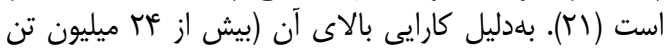

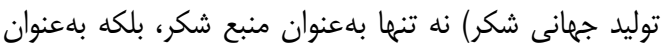

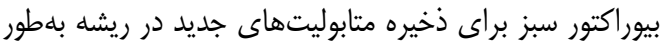

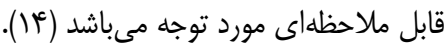

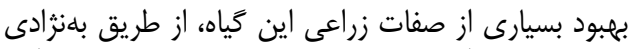

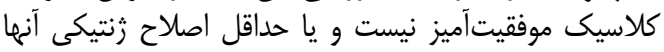

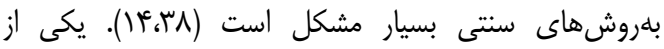

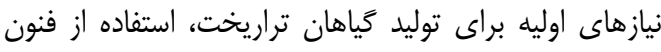

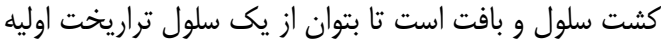

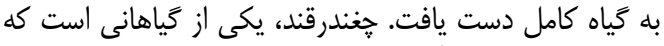

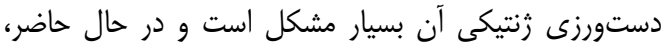

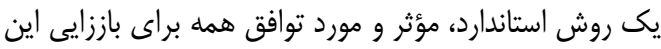

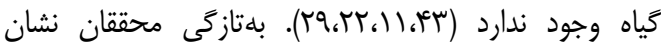

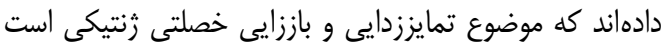

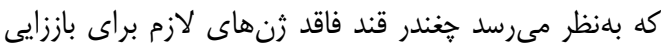

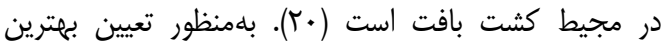

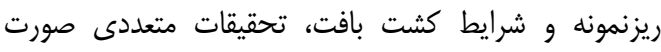

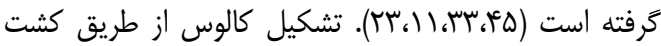

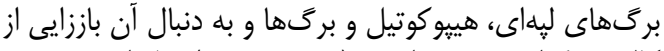

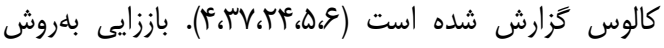

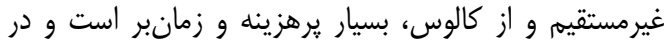

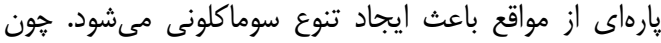

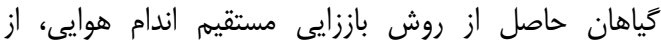


بعد از كذشت س روز، بذرها ريشهدار به محيط آب آنَار

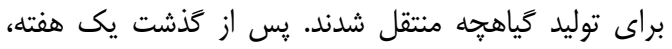

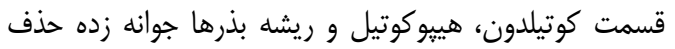

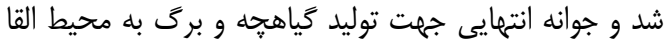
جوانه انتقال داده شد.

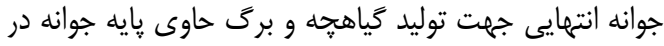

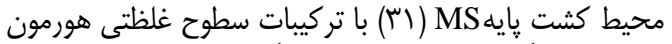
هاى BA

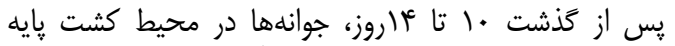
IBA با تركيبات هورمونى

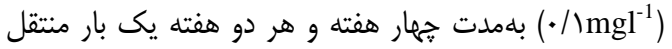

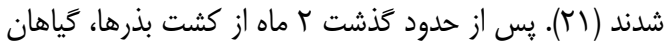

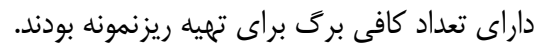

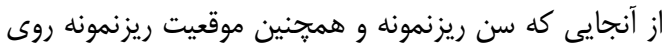

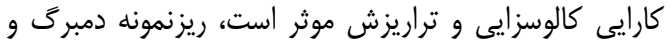

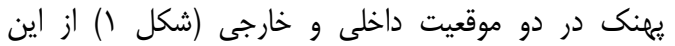

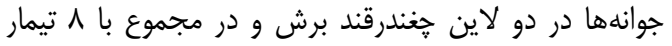

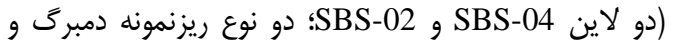

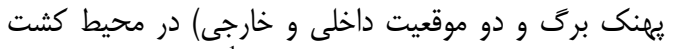

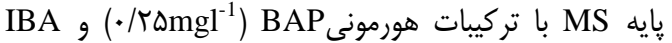

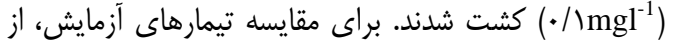

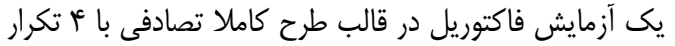

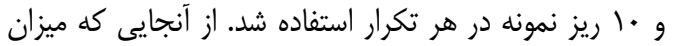

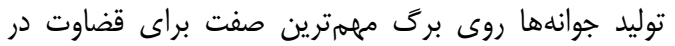

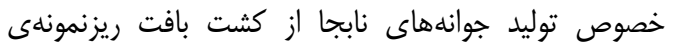

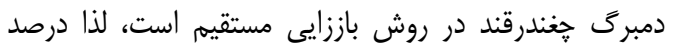

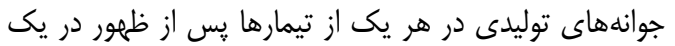

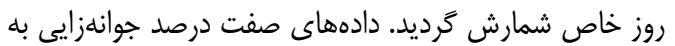

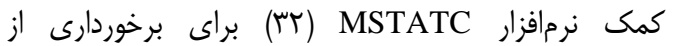

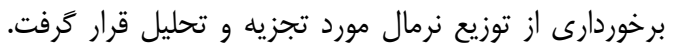

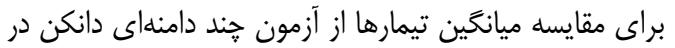
صطح معنى دارى مربوطه استفاده شد.
جوانهها بهدليل باززايى مستقيه و نيز به حداقل رسيدن تنوع

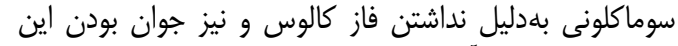

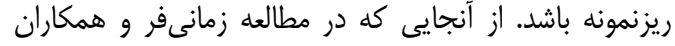

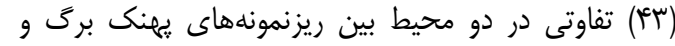

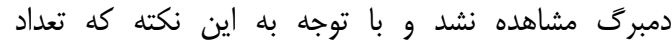

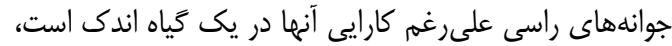

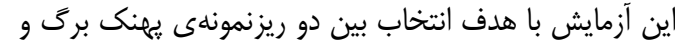

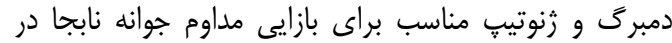

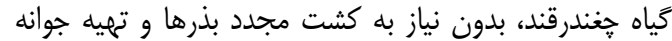

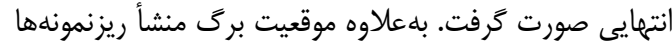

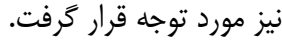

\section{مواد و روشها}

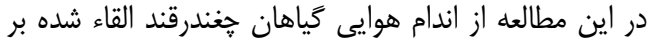

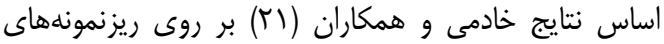

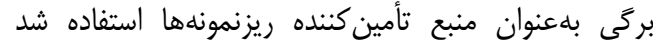

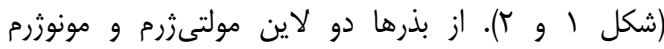

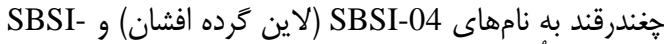

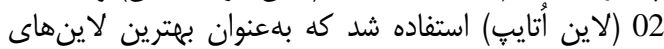

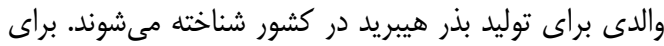

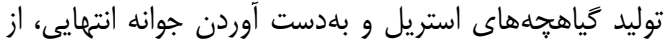
روش نوروزى و همكاران (سז) استفاده شد. در ابتدا، بذرها

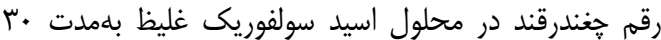

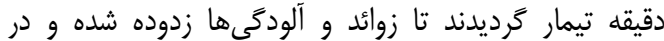

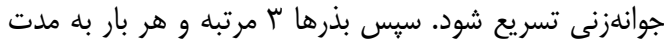

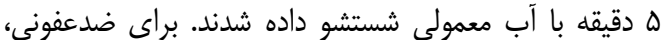

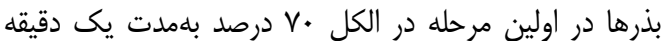

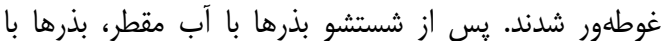
محلول هييوكلريت سديم

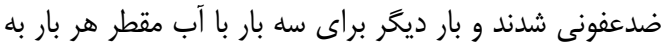

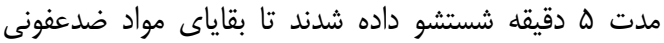

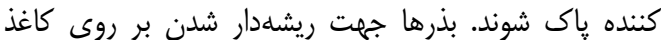
صافى استريل درون يترىديش در تاريكى قرار داده شدند.

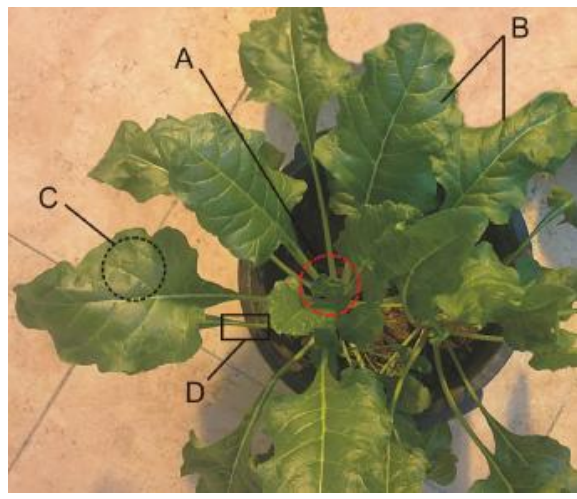

شكل (- محل قرارگيرى نسبى ريزنمونهاى مختلف: A) برى داخلى B (B) برى خارجى Cx) ريزنمونه يهنك D) ريزنمونه دمبرى

Figure 1. The relative position of different explants: A )Internal leaf and B) External leaf C) Leaf blade explant D) Petiole explant. 
داراى مقادير مناسب اكسين و سيتو كنين كشت مئشوند

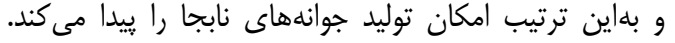

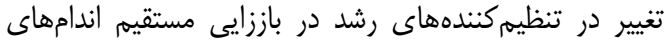

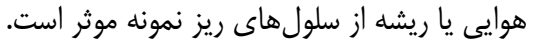

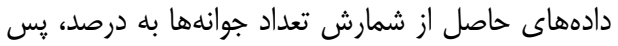

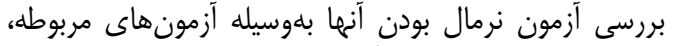

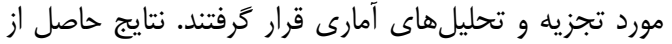

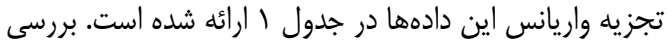

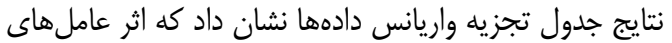

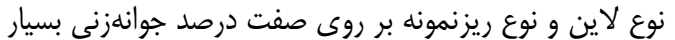

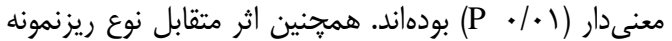

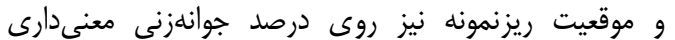

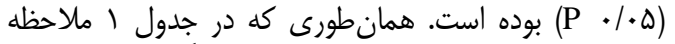

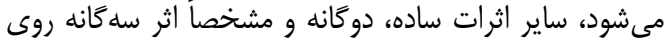
درصد جوانهزنى معنى سار نشدات سادها دواند.
نتايج و بحثث

يكى از روش هاى روت ريز ازديادى توليد جوانههاى نابجا است.

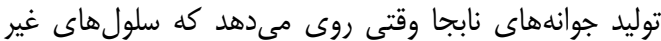

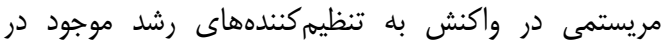

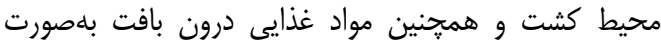

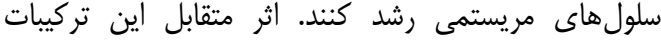
موجب توانايى باززايى شبه مريستمى (سلولهاى كارئ كه ساختار

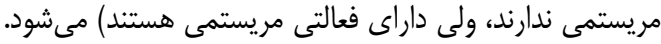

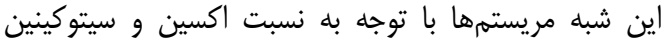

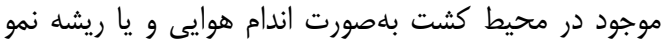

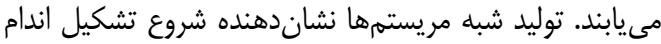

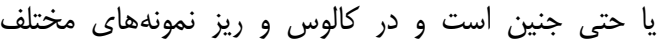

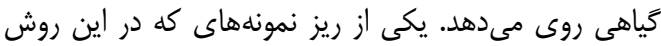

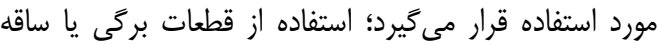

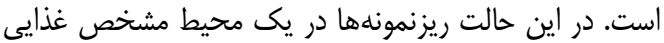

جدول ا- تجزيه واريانس صفت درصد جوانهاى توليدى حاصل از كشت بافت در خغندرقند Table 1. Analysis of variance for number of buds produced from tissue culture in sugar beet

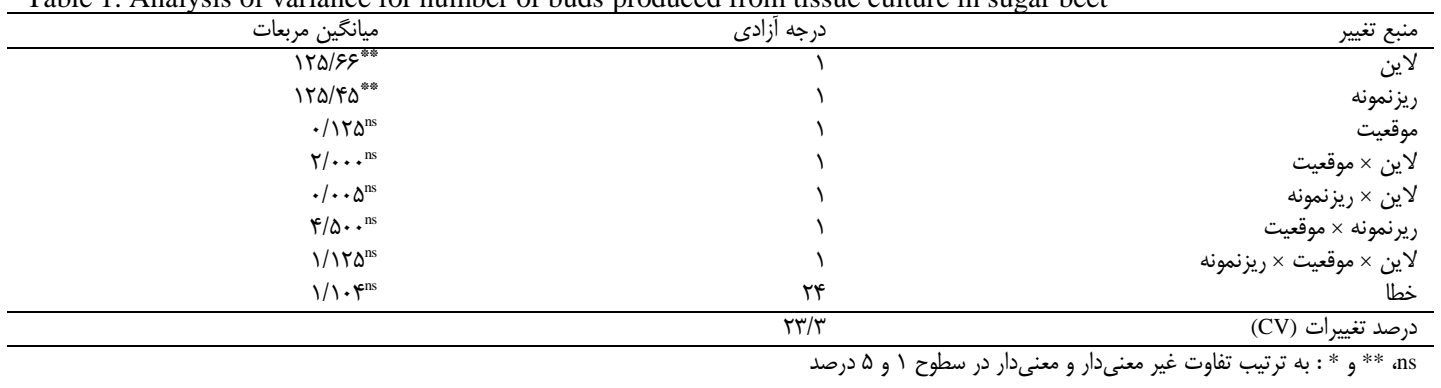

توليد بيشتر جوانه برترى دارد (جدول rابل). مقايسه ميانخين اثر

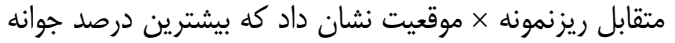

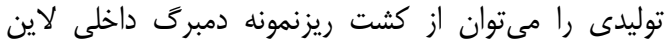

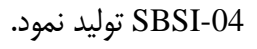

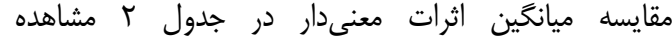

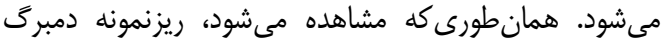

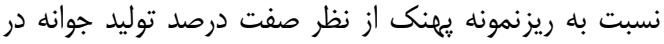

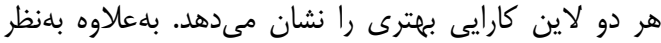

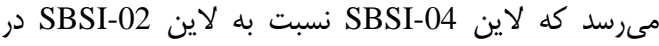

جدول r- مقايسه ميانگين صفت ميانگين درصد توليد جوانهها به روش دانكن Table 2. Mean analysis of the average number of generated buds, using Duncan multiple range test

\begin{tabular}{|c|c|c|c|}
\hline ميانكين درصد جوانها & كرو & لاين & موقعيت ريزنمونه \\
\hline$V / T \Delta$ & A & SBSI- $\cdot{ }^{r}$ & دمبرگ داخلى \\
\hline s/VQ & A & SBSI- $\cdot{ }^{f}$ & دمبركَ خارجى \\
\hline$\Delta / v \Delta$ & $\mathrm{AB}$ & SBSI- $\bullet f^{f}$ & يهنك خارجى \\
\hline$F / V Q$ & $\mathrm{BC}$ & SBSI- $\cdot Y$ & دمبرى داخلى \\
\hline$\varphi /$. & $\mathrm{C}$ & SBSI- $\bullet Y$ & دمبرى خارجى \\
\hline$\varphi /$. & $\mathrm{C}$ & SBSI- $\cdot F^{f}$ & يهنك داخلى \\
\hline $1 / V \Delta$ & D & SBSI- $\cdot Y$ & يِهنك داخلى \\
\hline I/VQ & D & SBSI- $\cdot Y$ & يهنك خارجى \\
\hline
\end{tabular}




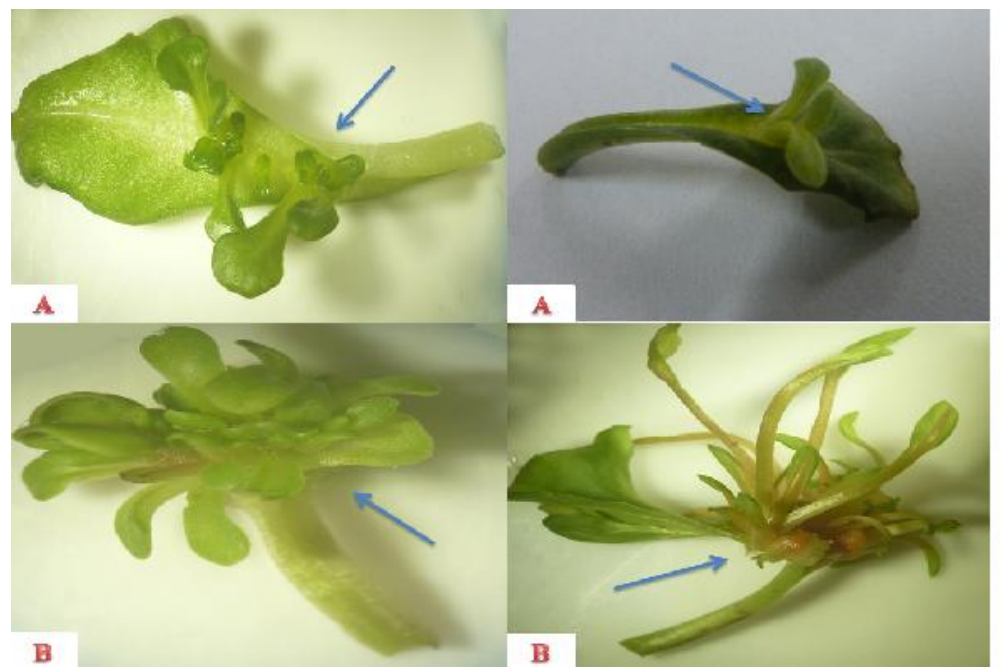

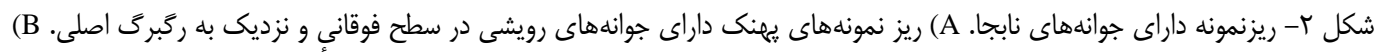

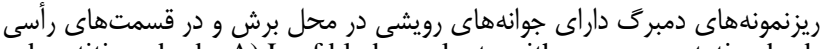

Figure 2. Explants with adventitious buds. A) Leaf blade explants with upper vegetative buds close to the main vein. B) Petiole explants with vegetative buds at the apical side and at cutting area

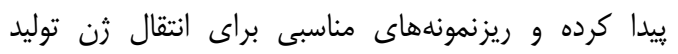

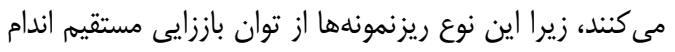

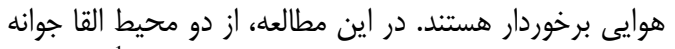

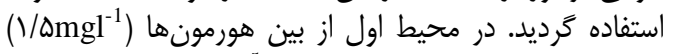

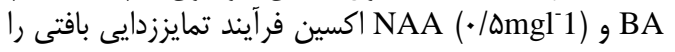

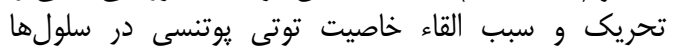

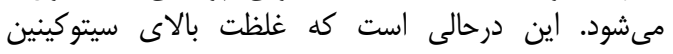

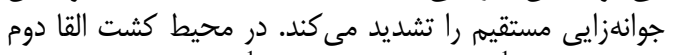

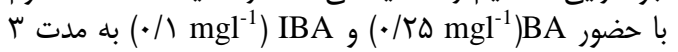

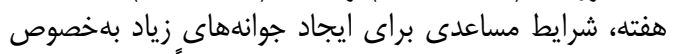

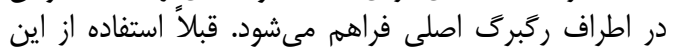

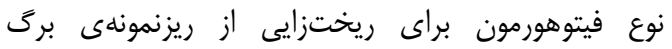

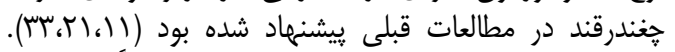

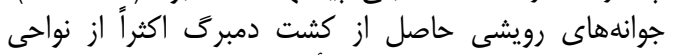

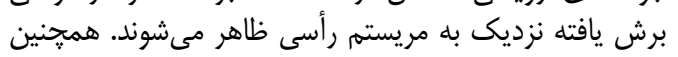

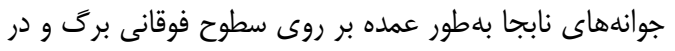

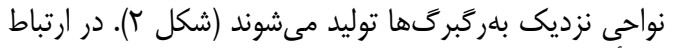

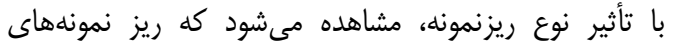

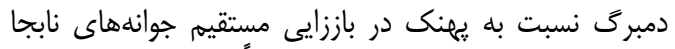

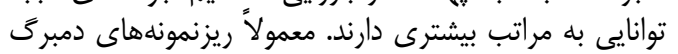

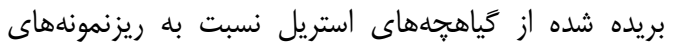

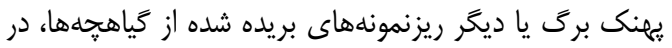

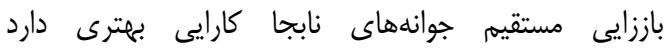

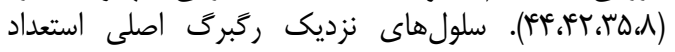

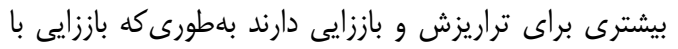

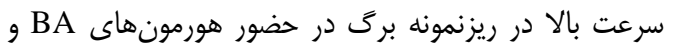

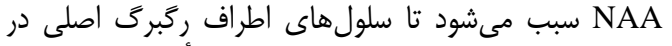

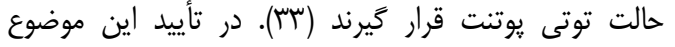

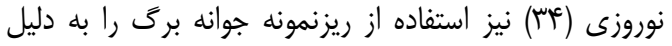
داشتن يك منبع دائمى توليدكننده اين ريزنمونهان اندا، توصيه
مطالعات كشتبافت هغندرقند در شرايط آزمايشكاهى براى إياء

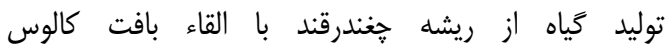

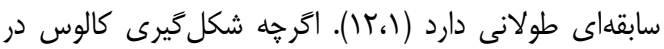

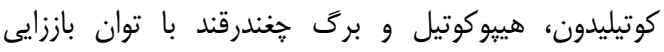

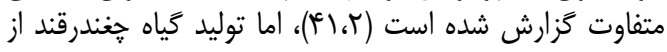

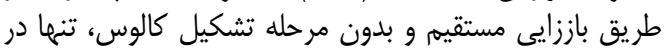

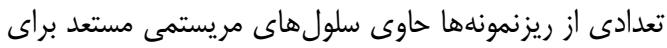

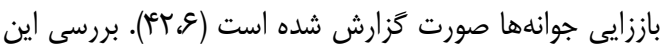

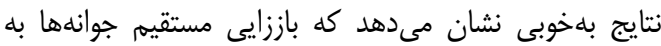

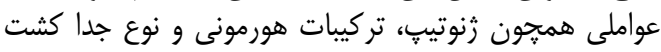

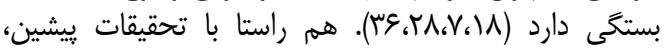

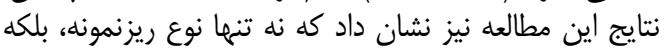

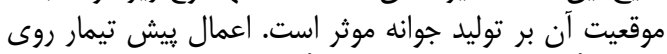

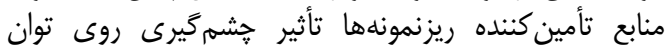

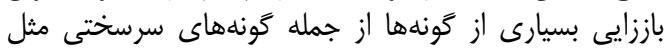

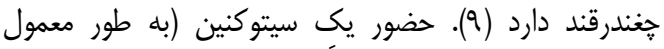

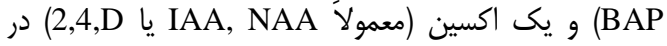

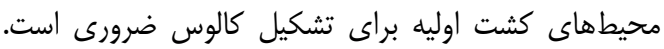

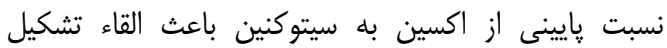

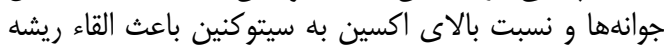

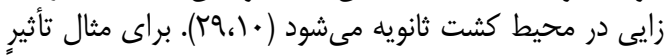

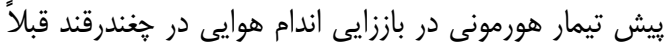

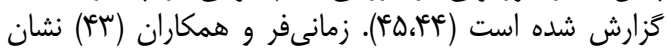

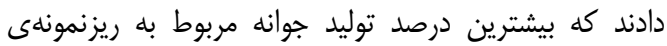

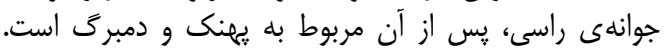

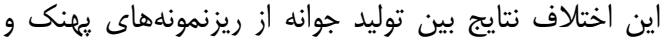

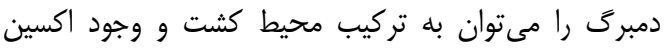

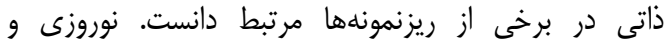

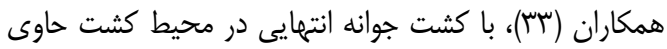

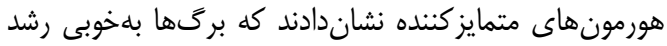


بيرونىتر، در بيدايش مستقيم جوانههاى نابجا باسخ بهترى

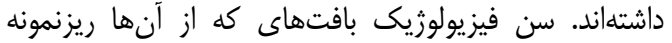

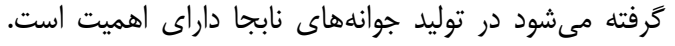

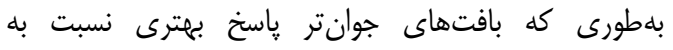

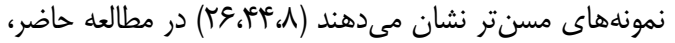

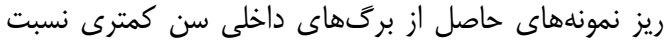

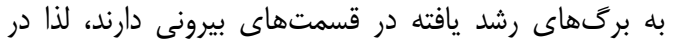

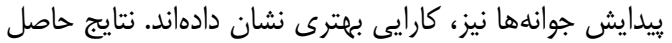

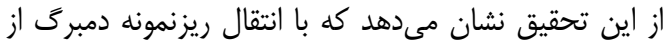

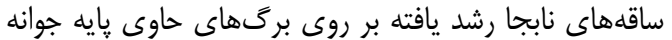
در روش باززايى مستقيم به محيط كشت رئ

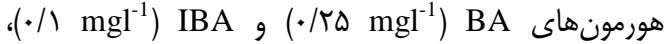

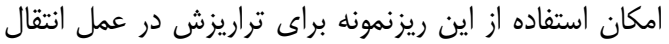

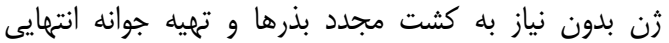

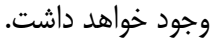

تشكر و قدردانى

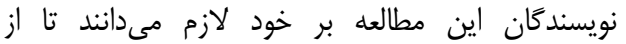

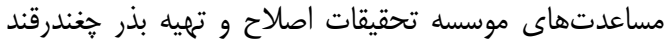

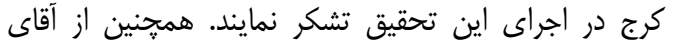

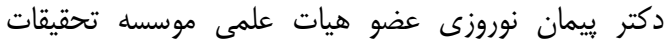
اصلاح و تهيه بذر تجندرقند كرج قدردانى به عمل مى آيد.

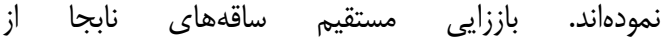

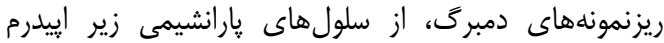

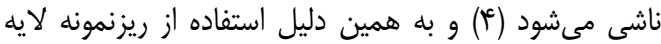

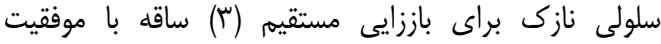
بالاترى همراه بوده است.

SBSI-04 نتايج مقايسه ميزان جوانهزنى در دو لاني

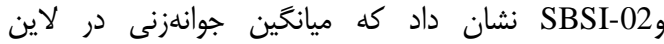

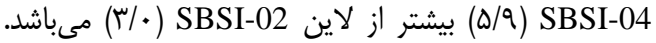

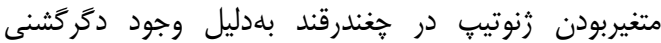

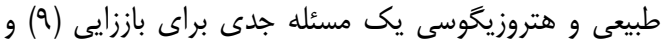

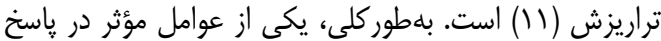

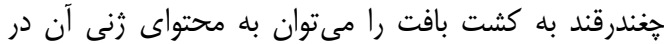

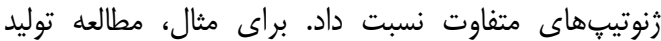

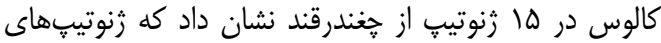

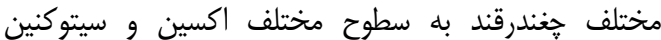

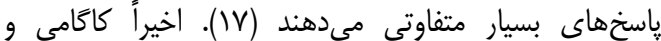

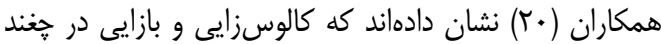

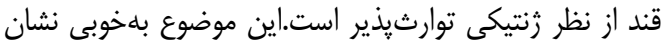

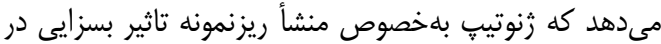

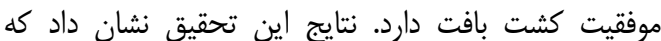

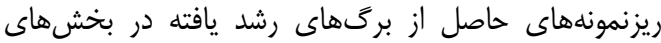

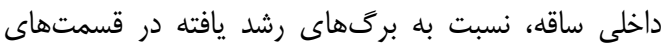
Butnko, R.G., A.I. Atanassov and V.V. Urmantseva. 1972. Some feature of sugar beet tissue cultures. Phytomorphology, 22: 140-143.

2. Catlin, D.W. 1990. The effect of antibiotics on inhibition of callus induction and plant regeneration from cotyledons of sugar beet (Beta Vulgaris L.). Plant Cell Reports, 9: 285-288.

3. Detrez, C., R.S. Sangwan and B.S. Sangwan-Norreel. 1989. Phenotypic and karyotypic status of Beta vulgaris plants regenerated from direct organogenesis in petiole culture. Theoretical and Applied Genetics, 77: 462-468.

4. Detrez, C., T. Tetu, R.S. Sangwan and B.S. Sangwan-Norreel. 1988. Direct organogenesis from petiole and thin cell layer explants in sugar beet cultured in vitro. Journal of Experimental Botany, 39: 917-926.

5. Dovzhenko, A. and H.U. Koop. 2003. Sugar beet (Beta vulgaris L.): shoot regeneration from callus and callus protoplasts. Planta, 217:374-381.

6. Freytag, A.H., S.C. Anand, A.P. Rao-Arelli and L.D. Owens. 1988. An improved medium for adventitious shoot formation and callus induction in Beta vulgaris L. in vitro. Plant Cell Reports, 7: 30-34.

7. Gosak, M. and M. Szota. 1992. Micropropagation of sugar beet (Beta vulgaris L.) Trisomics in vitro culture. Genetica Polonica, 33: 115-118.

8. Grieve, T.M., K.M.A. Gartland and M.C. Elliott. 1997. Micropropagation of commercially important sugar beet cultivars. Plant Growth Regulation, 21: 15-18.

9. Gürel, E., E. Topal and S. Gurel. 2003. The effect of pretreating seedlings with BAP on direct shoot regeneration from petiole explants of sugar beet (Beta vulgaris L). Asian-Pacific Journal of Biology and Biotechnology, 11: 57-62.

10. Gürel, E. 1997. Callus and root develonment from leaf explants of sugar beet (Beta vulgaris L.): variability between cultivars. Turkish Journal of Botany, 21: 131-136.

11. Hisano, H., Y. Kimoto, H. Hayakawa, J. Takeichi, T. Domae, R. Hashimoto, J. Abe, S. Asano, A. Kanazawa and Y. Shimamoto. 2004. High frequency Agrobacterium-mediated transformation and plant regeneration via direct shoot formation from leaf explants in Beta vulgaris and Beta maritima. Plant Cell Reports, 22: 910-918.

12. Hooker, M.P. and M.W. Nabors. 1977. Callus initiation, growth, and organogenesis in sugar beet (Beta vulgaris L.). Zeitschrift fur Pflanzen Physiologie, 84: 237-246.

13. Hussey, G. and A. Hepher. 1978. Clonal propagation of sugar beet plants and the formation of polyploidy by tissue culture. Annals of Botany, 42: 477-479.

14. Ivic-Haymes, S.D. and A.C. Smigocki. 2005. Biolistic transformation of highly regenerative sugar beet (Beta vulgaris L.) leaves. Plant Cell Reports, 23: 699-704.

15. Ivic-Haymes, S.D. and A.C. Smigocki. 2005. Identification of highly regenerative plants within sugar beet (Beta vulgaris L.) breeding lines for molecular breeding. In Vitro Cellular and Developmental BiologyPlant, 41: 483-488. 
16. Jacq, B., T. Tetu, R.S. Sangwan, A.D. Laat and B.S. Sangwan-Norreel. 1992. Plant regeneration from sugar beet (Beta vulgaris L.) hypocotyls cultured in vitroand flow cytometric nuclear DNA analysis of regenerants. Plant Cell Reports, 11: 329-333.

17. Jarl, C. and C.H. Bornman. 1986. Observations on genotypic variation in Beta vulgaris (sugar beet) tissues cultured in vitro. Hereditas, 105: 55-59.

18. Jianfeny, Z., L. Tianran and D. Xianglan. 1997. Highly efficient induction of sugar beet plant regeneration. Chinese Journal of Biothchnology, 13: 185-191.

19. Joersbo, M., S.G. Petersen and F.T. Okkels. 1999. Parameters interacting with mannose selection employed for the production of transgenic sugar beet. Plant Physiology, 105: 109-115.

20. Kagami, H., K. Taguchi, T. Arakawa, Y. Kuroda, H. Tamagake and T. Kubo. 2016. Efficient callus formation and plant regeneration are heritable characters in sugar beet (Beta vulgaris L.), Hereditas, 153: 12 pp.

21. Khademi, M. and F. nazarian-firouzabadi. 2014. The influence of two types of hormones (BA and NAA) on appearance of shoot base explants in sugar beet (Beta vularis L.). Agriculural Biotechnology, 1: 47-52.

22. Khwaje poor, M. 2007. Industrial Plants. $3^{\text {th }}$ edn. Jihad University unit Technology of isfahan, Iran, 580 (In Persian).

23. Kishchenko, E.M., I.K. Komarnitskii and N.V. Kuchuk. 2005. Production of transgenetic sugarbeet (Beta vulgaris L,) plants resistant to phosphinothricin. Cell Biology International, 29: 15-19.

24. Krens, F.A. and D. Jamar. 1989. The role of explant source and culture conditions on callus induction and shoot regeneration in sugarbeet (Beta vulgaris L.), Journal of Plant Physiology, 134: 651-655.

25. Krens, F.A., A. Trifonova, L.C.P. Keizer and R.D. Hall. 1996 .The effect of exogenously-applied phytohormones on gene transfer efficiency in sugar beet (Beta vulgaris L.). Plant Science, 116: 97-106.

26. Kuykendall, L.D., T.M. Stockett and J.W. Saunders. 2003. Rhizobium radiobacterconjugation and callusindependent shoot regeneration used to in-troduce the cercosporin export genecfpfromCercosporainto sugar beet (Beta vulgaris L,). Biotechnology Letters, 25: 739-744.

27. Lindsey, K. and P. Gallois. 1990. Transformation of sugar beet (Beta vulgaris L.) by Agrobacterium tumefaciens. Journal of Experimental Botany, 41: 529-536.

28. Mikami. T.. T. Kinoshita and H. Saito. 1985. Clonal Propagation of sugar beet plants by apical meristem culture. Journal of the Faculty of Agriculture, Hokkaido University, 62: 325-333.

29. Mikami, T., R.N. Sudoh and T. Kinoshita. 1989. Genotypic variation in the in vitro morphogenesis from leaf explants of Beta vulgaris L. and Beta maritima L. Euphytica, 40: 271-273.

30. Mishutkina, Y.V. and A.K. Gaponenko. 2006. Sugar beet (Beta vulgaris L.) morphogenesis in vitro: Effect of phytohormone type and concentration in the culture medium, type of explants, and plant genotype on shoot regeneration frequency. Russian Journal of Genetics, 42: 150-157.

31. Murashing, T. and F. Skoog. 1962. A revised medium for rapid growth and bioassay with tobacco tissue culture. Plant Physiology, 15: 473-497.

32. Nissen, D. 1989. MSTATC users guide. Michigan state university, 249 pp.

33. Norouzi, P., M.A. Malboobi, K. Zamani and B. Yazdi-Samadi. 2005. Using competent tissue for efficient transformation of sugerbeet (Beta vulgaris L.). In Vitro Cellular and Developmental Biology-Plant, 41: 1116.

34. Norouzi, P. 2012. Regeneration and transformation of haploid leaf and embryogenic tissues derived from ovule cultuer in sugar beet (Beta vulgaris L.). Journal of crop Breeding, 10: 63-79 (In Persian).

35. Ritchie, G.A., K.C. Short and M.R. Davey. 1989. In vitro shoot regeneration from callus, leaf axils and petioles of sugar beet (Beta vulgaris L.). Journal of Experimental Botany, 40: 277-283.

36. Sabir, A.A. and B.V. Ford-lioyd. 1991. Processing crop plant germplasm in vitro for mass production of regenerants: a case study with beet. Journal of Biotechnology, 17: 257-268.

37. Saunders, J.W. and W.P. Doley. 1986. One step shoot regeneration from callus of whole plant leaf explants of sugar beet lines and somaclonal variation of in vitro culture behaviour. Journal of Plant Physiology, 124: 473-479.

38. Saunders, J.W. and C.J. Tsai. 1999. Production of somatic embryos and shoots from sugar beet callus: Effects of abscisic acid, other growth regulators, nitrogen source, sucrose concentration and genotype. In Vitro Cellular and Developmental Biology-Plant, 35: 18-24.

39. Sharma, H.C., K.K. Sharma, N. Seetharama and R. Ortiz. 2000. Prospect for using transgenic resistance to insect in crop improvement. Journal of Biotechnology, 3: 76-95.

40. Snezana, D., I. Haymes and A.N.N.C. Smigocki. 2005. Identification of highly regenerative plants within sugar beet (Beta vulgaris L.) breeding lines for molecular breeding. In Vitro Cellular and Developmental Biology-Plant, 41: 483-488.

41. Snyder, G.W., J.C. Ingersoll and A.C. Smigocki. 1999. Introduction of pathogen Defense Genes and a Cytokinin Biosynthesis Gene into Sugar Beet (Beta Vulgaris L.) by Agrobacterium or Particle Bombardment. Plant Cell Reports, 18: 829-834

42. Toldi, O., G. Gyulai, J. Kiss, I. Tarns and E. Balazs. 1996. Antiauxin enhanced microshoot initiation and plant regeneration from epicotyl-originated thin layer explants of sugar beet (Beta vulgaris L.). Plant Cell Reports, 15: 851-854.

43. Zamanifar, M., F. Nazarian-Firouzabdi and A. Ismaili. 2016. Effect of two different cytokinin plant hormones on direct regeneration of diefferent sugar beet explant. Journal of crop Breeding, 19: 203-208 ( In Persian).

44. Zhang, C.L., D.F. Chen and M.C. Elliott. 2001. Thidiazuron-induced organogenesis and somatic embryogenesis in sugar beet (Beta vulgaris L.). In Vitro Cellular and Developmental Biology-Plant, 37: 305-310.

45. Zhong, Z., H.G. Smith and T.H. Thomas. 1993. In vitroculture of petioles and intact leaves of sugar beet (Beta vulgaris L.). Plant Growth Regulation, 12: 59-66. 


\title{
Adventitious Shoot Production using Petiole Explants through Direct Regeneration in Sugar Beet
}

\author{
Hossein Mazaheri Kohanestani ${ }^{1}$, Farhad Nazarian-Firozabadi ${ }^{2}$, Ahmad Ismaili $^{3}$ \\ and Mitra Khadami ${ }^{4}$
}

1, 3 and 4- Graduated M.Sc. Student, Associate Professor and Ph.D. Candidate, Faculty of Agricultural, Lorestan University

2- Professor, Faculty of Agricultural, Lorestan University, (Corresponding author: nazarian.f@lu.ac.ir) Receive: September 30, 2016

Accepted: May 24, 2017

\begin{abstract}
Sugar beet (Beta vulgaris L.) is one of the two very important sugar crops in the world. Transgenic experiments, including tissue culture practices play a pivotal role in the investigation of the any gene function as well as introducing new functionally important genes. Tissue culture is inevitably required to employ any transgenic methods for making transgenic plants. Therefore, to find an appropriate explant to produce adventitious shoots in recalcitrant sugar beet plants without continuous seed germination, a completely randomize design (CRD) was carried out in a factorial arrangement. The adventitious shoots produced on leaves through direct regeneration method, were used as in vitro explant. Petiole and leaf disc explants were studied in two positions, located close and far from terminal buds in two sugar beet lines (SBSI04, SBSI-02). After four weeks and two consecutive sub-culturing in MS basal medium supplemented with IBA $\left(0.1 \mathrm{mgl}^{-1}\right)$ and BAP $\left(0.25 \mathrm{mgl}^{-1}\right)$ hormones, the number of shoots were counted and compared as percentage. Results of this study showed that the petiole explant produced the highest number of shoots. Furthermore, the number of the shoots was higher in SBSI-04 line than SBSI-02 line. Interestingly, the position of leaf explants had a significant effect on the number of shoots produced. Interior petiole explants, produced more shoots in SBSI-04 line as compared to the leaves farther than terminal buds, suggesting for producing transgenic sugar beet plants, interior petiole explants are recommended.
\end{abstract}

Keywords: Direct Regeneration, Explants, Growth hormones, Petiole, Transformation 